\title{
Péricardite constrictive accompagnée de calcification péricardique
}

Kevin R. An MD, Steve K. Singh MD MSc

- Citation : CMAJ 2021 June 7;193:E853. doi : 10.1503/cmaj.202346-f

Voir la version anglaise de l'article ici : www.cmaj.ca/lookup/doi/10.1503/cmaj.202346

$\mathbf{U}$ n homme de 71 ans avec un antécédent de mélanome localement réséqué s'est présenté chez son médecin de famille avec un œdème bilatéral aux membres inférieurs en progression depuis 2 ans, une ascite, une perte de poids, de la fatigue et de la dyspnée à l'effort. Ses antécédents médicaux comprenaient aussi une maladie pseudogrippale chronique, contractée 3 ans auparavant. Dans le cadre d'un bilan pour la récurrence du mélanome, qui était par ailleurs sans particularités, une tomodensitométrie thoracique a décelé des calcifications péricardiques et épicardiques, compatibles avec un diagnostic de péricardite constrictive (figure 1A). Une échocardiographie transthoracique a démontré une effusion péricardique, un épaississement du péricarde, un rebond septal et une dilatation de la veine cave inférieure. Le cathétérisme cardiaque a révélé une égalisation de la pression diastolique dans les 4 cavités à 20 (plage normale 2-12) mm Hg, confirmant une péricardite constrictive.

Une péricardectomie a révélé des dépôts calciques blancs sur le cœur; nous avons réséqué le péricarde antérieur jusqu'au nerf phrénique de façon bilatérale pour soulager la constriction cardiaque (figure 1B). L'examen pathologique n'a montré aucun signe de malignité et les cultures d'échantillons n'ont pas révélé de traces de tuberculose. Bien que la causalité ne peut être déterminée en toute certitude, nous considérons qu'il est fort probable que la péricardite constrictive était une séquelle à long terme d'une infection virale. Les symptômes de distension abdominale, d'enflure des membres inférieurs, de fatigue et d'essoufflement présentés par le patient se sont améliorés de façon constante après l'intervention et celui-ci a obtenu son congé 2 semaines après la chirurgie.
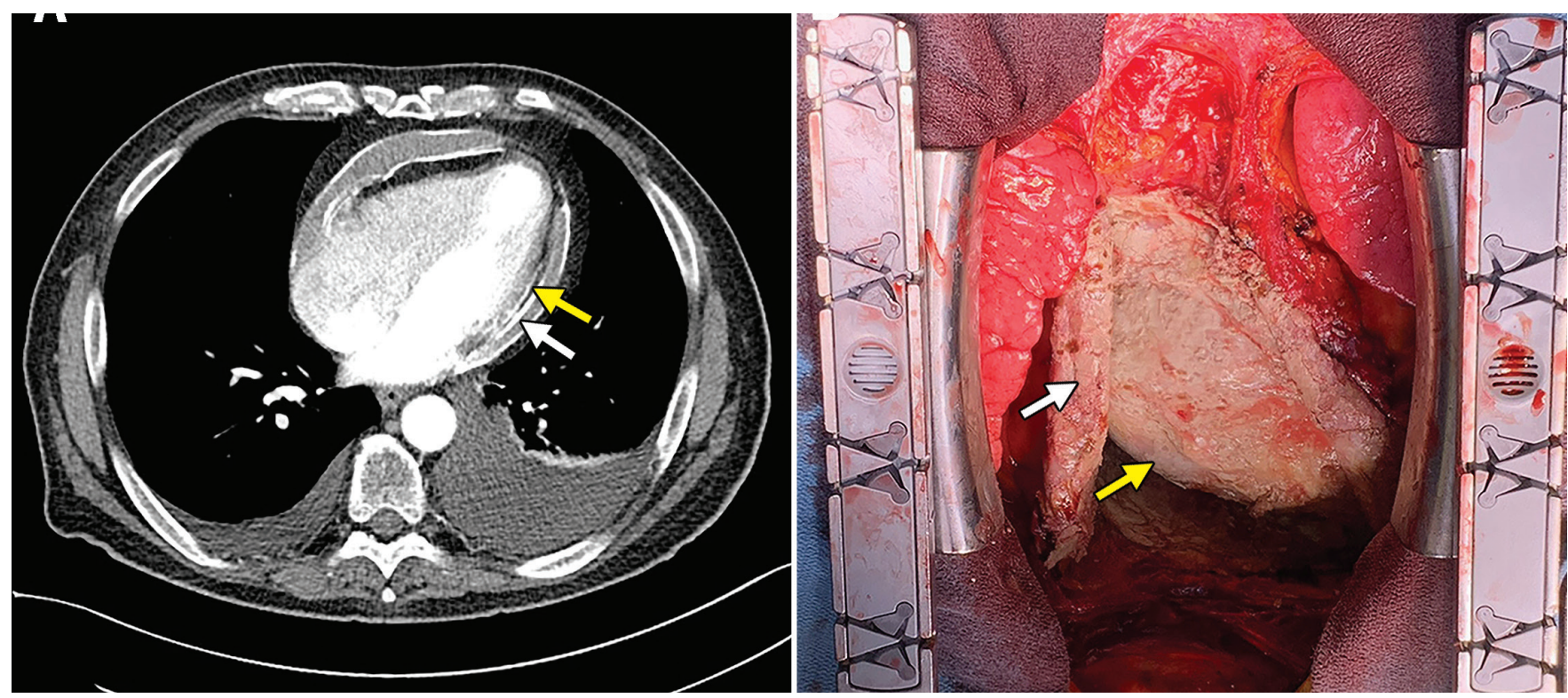

Figure 1 : Péricardite constrictive chez un homme de 71 ans. A) Tomodensitométrie avec agent de contraste de la cage thoracique d'un patient montrant une calcification périphérique du péricarde (flèche blanche) et de l'épicarde (flèche jaune). B) Image peropératoire des dépôts calciques blancs du cœur du patient, montrant le péricarde (flèche blanche) et l'épicarde (flèche jaune). 
La péricardite constrictive est une affection inflammatoire chronique du péricarde dont l'incidence est de 1 cas sur 100000 personnes aux États-Unis ${ }^{1}$. Elle est souvent induite par une péricardite idiopathique ou virale, une chirurgie cardiaque antérieure, une irradiation médiastinale ou la tuberculose; on croit qu'elle serait déclenchée par une réponse immunitaire, menant à l'apparition d'un péricarde fibreux ou calcifié ${ }^{1,2}$. Dans le cadre d'une étude, des examens pathologiques ont montré que $28 \%$ des échantillons de péricardes réséqués étaient grossièrement calcifiés ${ }^{3}$. Les symptômes sont comparables à ceux d'une insuffisance cardiaque et il est difficile de distinguer cette affection d'une cardiomyopathie restrictive ${ }^{2}$. Le cathétérisme cardiaque bilatéral est le test diagnostique de référence ${ }^{4}$ et la prise en charge définitive est la péricardectomie.

\section{Références}

1. Mori M, Mullan CW, Bin Mahmood SU, et al. US national trends in the management and outcomes of constrictive pericarditis: 2005-2014. Can J Cardiol 2019;35:1394-9.

2. Syed FF, Schaff HV, Oh JK. Constrictive pericarditis - a curable diastolic heart failure. Nat Rev Cardiol 2014;11:530-44.

3. Oh KY, Shimizu M, Edwards WD, et al. Surgical pathology of the parietal pericardium: a study of 344 cases (1993-1999). Cardiovasc Pathol 2001;10:157-68.

4. Welch TD, Oh JK. Constrictive pericarditis. Cardiol Clin 2017;35:539-49.

\section{Intérêts concurrents : Aucun déclaré.}

Cet article a été révisé par des pairs.

Les auteurs ont obtenu le consentement du patient.

Affiliations : Division de chirurgie cardiaque, Partenaires de santé Trillium, Université de Toronto, Toronto, Ont.

Propriété intellectuelle du contenu : Il s'agit d'un article en libre accès distribué conformément aux modalités de la licence Creative Commons Attribution (CC BY-NC-ND 4.0), qui permet l'utilisation, la diffusion et la reproduction de tout médium à la condition que la publication originale soit adéquatement citée, que l'utilisation se fasse à des fins non commerciales (c.-à-d., recherche ou éducation) et qu'aucune modification ni adaptation n'y soit apportée. Voir : https://creativecommons.org/licenses/by-nc-nd/4.0/deed.fr.

Correspondance : Steve Singh, steve.singh@thp.ca

Les images cliniques sont choisies pour leur caractère particulièrement intéressant, classique ou impressionnant. Toute soumission d'image de haute résolution claire et bien identifiée doit être accompagnée d'une légende aux fins de publication. On demande aussi une brève explication (300 mots maximum) de la portée éducative des images, et des références minimales. Le consentement écrit du patient au regard de la publication doit être obtenu avant la soumission. 